\title{
Application of Chalcogenide Fibers
}

\author{
D.W. Hewak, K. Khan and C.C. Huang \\ Optoelectronics Research Centre, University of Southampton, Southampton, United Kingdom SO17 1BJ \\ dh@orc.soton.ac.uk
}

\begin{abstract}
Chalcogenide glass optical fibers have been extensively studied since 1967, when sulphide based fibers and their potential applications were first proposed. While high quality fiber drawn from alloys containing a variety of chalcogen elements have been realized, their delicate nature, complicated fabrication methodology and expense has restricted widespread application and commercial acceptance. In this paper we describe our current work on the fabrication and application of chalcogenide fiber and our vision for their practical implementation in the future.
\end{abstract}

OCIS codes: (060.2390) Fiber optics, infrared, 060.2280 (Fiber design and fabrication), (060.2290) Fiber materials

\section{Introduction}

Chalcogenide glasses based on sulphur, selenium and/or tellurium provide a wide range of materials with sufficient stability and transmission for optical fiber applications at infrared wavelengths. Some of the earliest demonstrations of chalcogenide fiber were with the simple binary glass arsenic sulphide, which provided a broad glass formation region and transmission across the mid infrared [1]. Subsequent work moved to selenide and telluride glasses, which provided transmission at longer wavelengths. However, even today the manufacturing of chalcogenide glass and fiber is not straightforward and continues to rely on conventional melt quench techniques to form the glass, whether it is drawn from a solid preform or remelted and drawn using crucible techniques. Most commercial fiber today has a loss between 0.1 and $1.0 \mathrm{~dB} / \mathrm{m}$, far from the intrinsic loss limit [2]. Silica optical fiber depends critically on chemical vapour deposition (CVD) techniques to achieve losses that are several orders of magnitude lower than another other glass drawn into fiber form. To date, CVD techniques have not been implemented in chalcogenide fiber fabrication [3], yet they have the potential to radically improve the manufacturing process. The work describes here is the first phase, within a five year project, to adapt current fibre manufacture with novel chemical vapour deposition chemistry for application in the next generation of chalcogenide fiber.

\section{Glass Fabrication}

Our work is currently focused on germanium sulphide based glass, which we have fabricated directly by CVD deposition in the form of thin films and as a bulk glass. Analysis shows a high-purity amorphous material, which has high potential for the fabrication of the chalcogenide optical waveguides. In the process, we achieve germanium sulphide through reaction of $\mathrm{GeCl}_{4}$ and $\mathrm{H}_{2} \mathrm{~S}$ at $450-600^{\circ} \mathrm{C}$ with impurity levels measured to be on the order of parts per billion, a factor of one hundred better than that achieved by melting refractory raw materials [4].

\section{Fibre Drawing}

The second phase of our work is developing fiber drawing processes compatible with the CVD technology now demonstrated. We will revisit crucible drawing technology first established over twenty years ago and adapt this to the requirements of today's optical fiber requirements.

\section{References}

[1] J. Sanghera and I. Aggarwal, Infrared Fiber Optics, CRC Press, Boca Raton, FL (1998).

[2] D.J.Brady, T.Schweizer, J.Wang, D.W.Hewak, "Minimum loss predictions and measurements in gallium lanthanum sulphide based glasses and fibre”, Journal of Non-Crystalline Solids 1998 Vol.242 pp.92-98

[3] J. Harrington, Infrared Fibers and Their Applications, SPIE Press, Bellingham, Washington USA (2004).

[4] C.C.Huang, D.W.Hewak, "High purity germanium sulphide glass for optoelectronic applications synthesized by chemical vapour deposition", Electronics Letters 2004 Vol.40(14) pp.863-865

\section{Acknowledgements}

This work is funded by the EPSRC Centre for Innovative Manufacturing in Photonics under EPSRC Reference EP/H02607X/1. Thanks are due to Professor D Payne, Principle Investigator and Dr. W Loh, IMRC Director for their support and to Mr. M Lessey, Edwin Weatherby Esq. and Mr. N Fagan for technical assistance. Laboratory management was overseen by Mr J. Tucknott and Mr. T Austin provided electronics support. 